\title{
CAVERNOUS PULMONARY TELANGIECTASIS
}

\author{
BY \\ JOHN HAYWARD AND LYNNE REID* \\ From the Royal Melbourne Hospital, Melbourne, Australia
}

A cavernous pulmonary telangiectasis is a wide peripheral communication between a dilated, tortuous, thin-walled pulmonary artery and the corresponding pulmonary veins. It is part of a more or less generalized condition of telangiectasia in which small telangiectases are scattered in variable distribution and numbers in the lungs and elsewhere in the body. The striking appearance and obvious clinical effects of the large pulmonary lesion have blinded observers to its relationship to the generalized telangiectasia.

The first report of this unusual condition was made by Bowers (1936), who found multiple "haemangiomatous" masses in the lungs of a baby boy who had died two days after birth from haemorrhage into the pleural cavity. The second case, also diagnosed at necropsy and reported by Rodes (1938), was that of a man aged 25 who had died of haemoptysis. There were multiple "haemangiomata" in the right lower and middle lobes and in the left upper lobe. In this case the chief clinical features, namely, dyspnoea, cyanosis, clubbing of the fingers, and polycythaemia, were described for the first time.

In the following year Smith and Horton (1939) recorded the first successful diagnosis of the condition during life, and named it "arteriovenous fistula of the lung." Since then it has been called cavernous haemangioma or arteriovenous fistula.

The next advance came when Shenstone (1942) described his successful removal of the lesion by pneumonectomy in 1940. The patient was a woman aged 23 whose signs and symptoms have been recorded by Hepburn and Dauphinee (1942). These authors directed attention to the fact that symptoms such as tinnitus, faintness, and thick speech which were seen in this case were attributable to the polycythaemia rather than directly to the abnormal anastomosis, and they noted the disappearance of the polycythaemia after the removal of the lesion.

A further step was the successful local excision of two haemangiomata from the right lung, followed at a second stage by resection of one from

\footnotetext{
*Research Fellow, National Health and Medical Research Council.
}

the left lung by Janes (1944). Since then approximately 25 more cases have been reported in full or mentioned in discussions, and Maier and his colleagues (1948) have recently made a notable addition to our knowledge by a study of the physiological responses to an arteriovenous shunt in the lung; but little thought seems to have been given to its pathology. Doubt about the pathology is responsible for the confusion in the naming of the condition.

Our interest was recently stimulated by encountering a patient who happens to be the oldest yet reported with the lesion. We wish to report this case, discuss the condition with special reference to its pathology and pathogenesis, and give our reasons for advocating the new name which forms the title of this paper.

\section{CASE Report}

A single woman, aged 52 years, was admitted to the Royal Melbourne Hospital on Dec. 15, 1947, with a seven months' history of precordial pain and vomiting once or twice daily for the previous two weeks. The vomiting bore no relation to the pain, which usually began two to three hours after a meal and was relieved by food. She gave a further history that although she had been generally pallid as a child her lips and fingers were always blue, and she could never do as much as her companions because of shortness of breath.

Her periods, which had always been scanty, ceased when she was 50 , and, for 20 years before this, nose bleeds had constantly preceded them by about 36 hours. During these years she had frequent attacks of migraine characterized by visual aura, vomiting, and headache. She admitted slight cough in the winter for some years, but any sputum she had produced had never been blood-stained.

She had never had any serious illness and had earned her own living throughout her life as a manageress of an hotel dining-room, but, because of her dislike of the cold, she had gone home for several months during each winter.

She was a plump woman with deeply cyanosed lips and fingertips although her skin elsewhere was very pale. One small naevus was present on the dorsum of the right hand and there was slight clubbing of her fingers. 


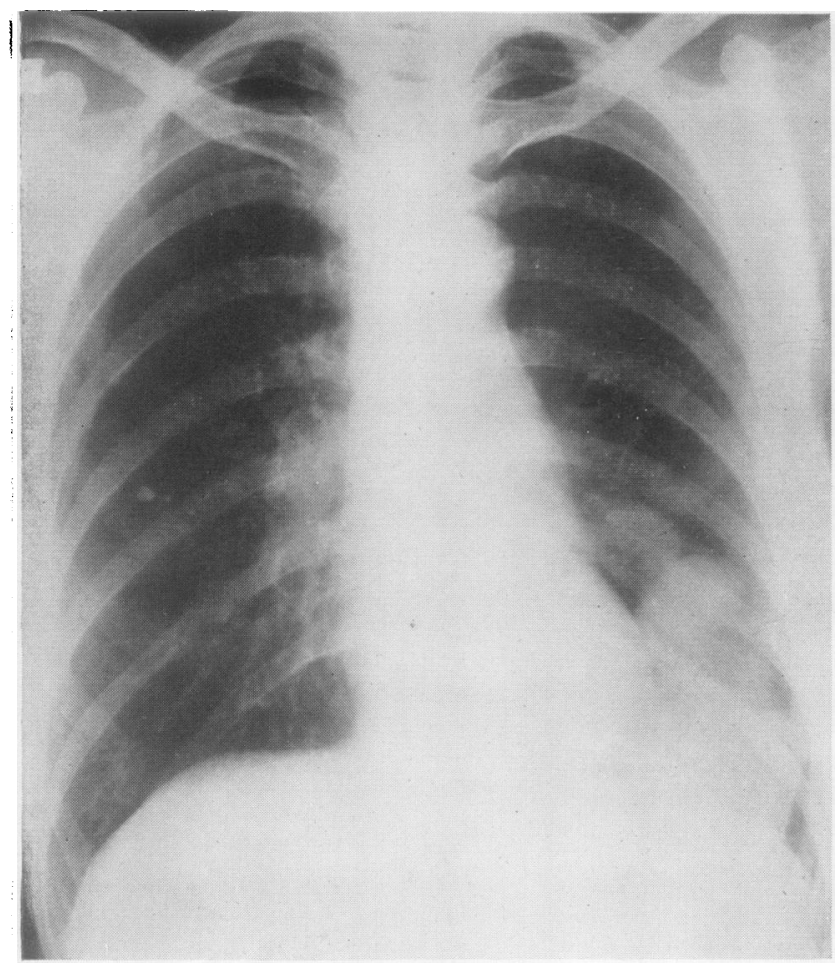

FIG. 1.-Postero-anterior radiograph showing large convoluted opacity extending from left hilum to left base. The small rounded shadow overlying the right hilum is less obvious.
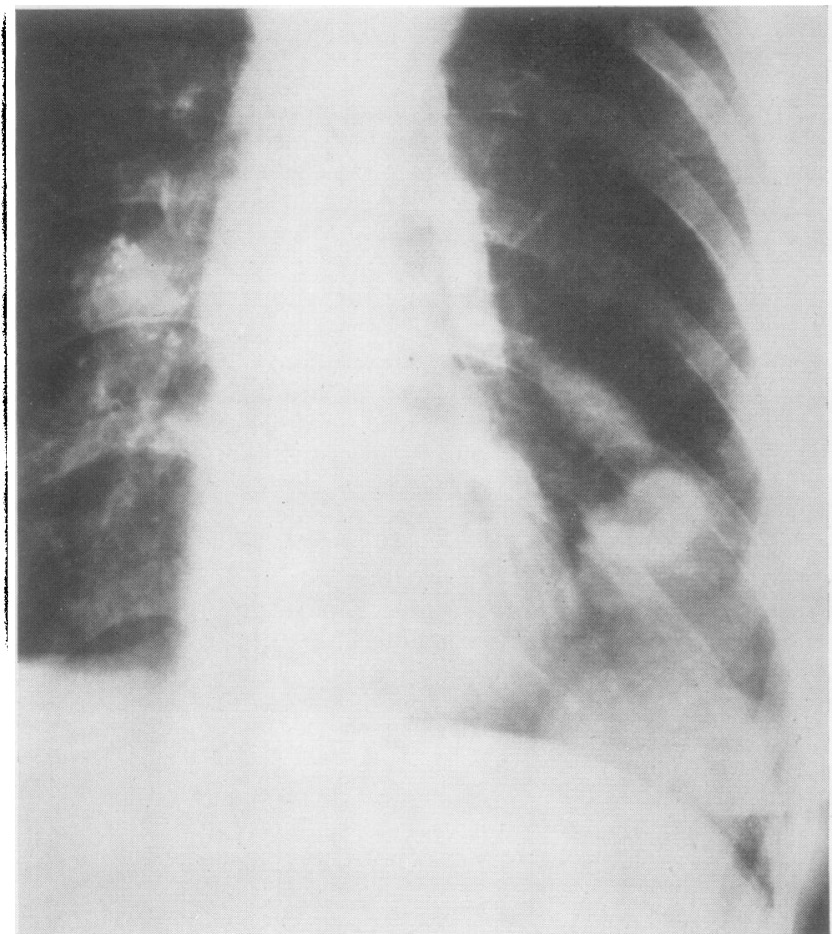

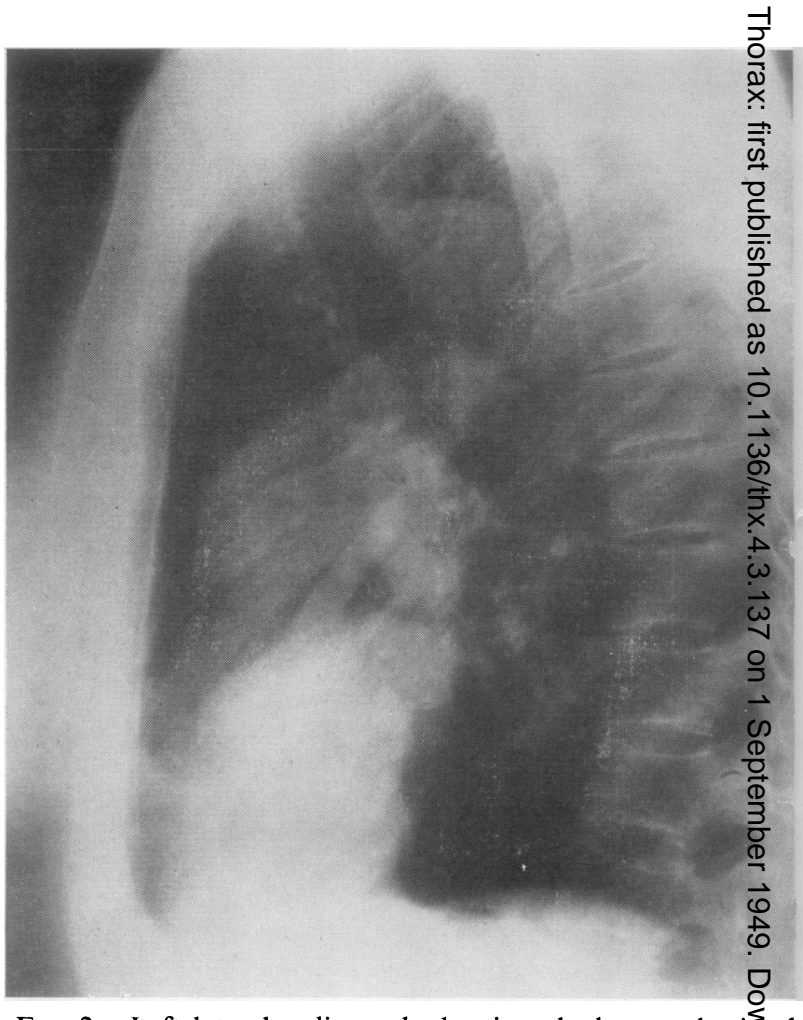

FIG. 2.-Left lateral radiograph showing shadow to be i th lingular area. The straight upper vein running to the oflu. and irregular shadow below it produced by the tortuous fitel overlying the lower vein are clearly seen. (CompareDwi) Fig. 7.)

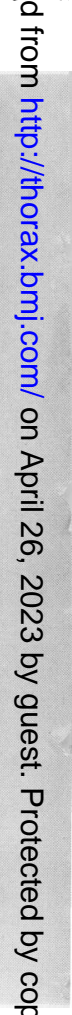

FIGS. 3 AND 4.-Postero-anterior radiographs taken in full inspiration (Fig. 3) and full expiration (Fig. 4) to show the large fluctutic in size of the shadow which is diagnostic of the condition. This variation in size with respiration was even more obvious $\overline{\bar{m}}^{-} \mathrm{tl}$ fluoroscopic screen. 
The heart sounds were normal and there was no murmur audible over the chest. The liver and spleen were not palpable. Her blood pressure was $140 / 95 \mathrm{~mm}$. of mercury, the pulse rate 76 per minute, and the respirations when at rest 18 per minute. Examination of the urine revealed nothing abnormal.

The report on her blood count was as follows:

Haemoglobin, $150 \%$ (i.e., 21 g. $/ 100 \mathrm{ml}$.).

Erythrocytes, 7,800,000/c.mm.

Colour index, 0.98 .

Leucocytes, $5,800 /$ c.mm.

Blood films, including differential count, were within normal limits. Because of the low leucocyte count the polycythaemia was considered to be secondary.

The radiological report read as follows:

"Calcified nodule in the right midzone; calcification of the tracheo-bronchial lymph glands, and of the right hilar region. Large ill-defined somewhat lobular opacity in the anterior and lower parts of the left lung."

The fractional test meal showed a high acid curve and some occult blood was demonstrated in the faeces. Her dyspeptic symptoms were regarded as due to an ulcer, but the significance of the polycythaemia and the pulmonary shadow was not appreciated.

As her dyspepsia had improved she chose to go home for Christmas and was advised to return later.

She was urgently readmitted on Feb. 9, 1948, immediately after an attack of substernal pain and vomiting which was associated with marked weakness and prostration. Her haemoglobin had fallen to $102 \%$ and a barium meal showed some pylorospasm with irregularity at the base of the duodenal cap. There is little doubt that these urgent symptoms were the result of haemorrhage from peptic ulceration secondary to the polycythaemia (Bockus, 1944).

She was then seen by us in consultation and a tentative diagnosis of cavernous pulmonary telangiectasis was made. This was supported by further radiological examination which showed that the tumour was larger in inspiration than expiration.

The oxygen saturation of the arterial blood was $64.1 \%$ of normal (i.e., $100 \mathrm{ml}$. of blood combined with $15.4 \mathrm{ml}$. of oxygen) when the haemoglobin was $125 \%$ (i.e., 17.5 g. haemoglobin), the level to which it had risen in the eight weeks following her readmission. The blood was collected under paraffin by femoral artery puncture and the estimation made by van Slyke's method. Her liver function tests were within normal limits. The patient agreed to submit to the operation.

On May 15 under pentothal, curare, and cyclopropane anaesthesia administered by Dr. Margaret McClelland a left postero-lateral transcostal thoracotomy was done through the bed of the sixth rib. There were a few loose adhesions over the lingula in which the lesion could be seen and felt as a vascular tumour extending from the hilum to the periphery of the lobe, pulsating slightly but giving no palpable thrill until partly compressed between the fingers. The normal-looking aerated lung tissue of the lingula surrounded the lesion proximally and terminated distally and below it in .a small pedunculated projection. Proximally the lesion bulged through the lung irregularly, simulating a lobulated appearance. Distally it came completely to the surface above the tip of aerated lung in a large ovoid, smooth, thinwalled sac covered only by visceral pleura. The dark blue blood it contained could be seen through its wall. Apart from the lesion in the lingula the left lung appeared normal.

The lingula was excised. The difficulty of dissection at the hilum was increased by the partial absence of a fissure between the lingula and lower lobe. Two large lingular veins, one about $\frac{1}{2}$ in. and the other about $\frac{1}{4}$ in. in diameter, and two lingular arteries, one huge and thin-walled, and the other normal in size, were ligated and divided. The bronchus with its bronchial arteries appeared normal ; it was divided, the stump closed with interrupted linen sutures, and the lingula stripped from the upper lobe in the usual way. The chest was closed after resecting a small portion of the tenth rib and inserting a drainage tube connected to an underwater seal.

We usually give a blood transfusion of two or three pints during lobectomy, but in this patient because of the polycythaemia only one pint was given during the operation, followed by two litres of glucose saline in the next 12 hours.

Convalescence was uneventful. The drainage tube was removed on the third day. Forty-three ounces of blood-stained serous fluid had drained through it. The patient sat out of bed on the sixth day. From the moment of ligation of the lingular vessels her colour improved and this was most noticeable on exertion. For instance, on the morning after operation coughing made her face and lips a brighter pink instead of more cyanosed as had been the case before operation.

During the 28 days following operation there was a progressive fall in the haemoglobin to $82 \%$ (R.B.C. $4,500,000$; C.I., $90 \%$ ). The oxygen saturation had increased to $92 \%$ of normal (i.e., $100 \mathrm{ml}$. of blood combined with $14.5 \mathrm{ml}$. of oxygen when the haemoglobin was $82 \%$-i.e., 11.5 g. haemoglobin). A naevus on her nasal septum was cauterized.

Since operation there has been no more dyspnoea on exertion than would be expected in a woman of her age, and she is living a normal life. The symptoms of peptic ulceration have vanished. The change in her character has been as striking as her physical improvement ; before operation she seemed a listless, rather weak, ineffectual sort of person, and was prone to tears. This was presumably due to cerebral anoxaemia because now she is a bright, determined, and quite impressive little woman.

Pathology.-Immediately after operation the bronchi of the specimen were injected with formalin until the lung substance was distended to its normal size, and the specimen was allowed to fix for two weeks. The large vessels and sac were distended with gelatin to maintain their shape, and when it had solidified the specimen was dissected to show the relations of the blood vessels (Figs. 5 and 6). 


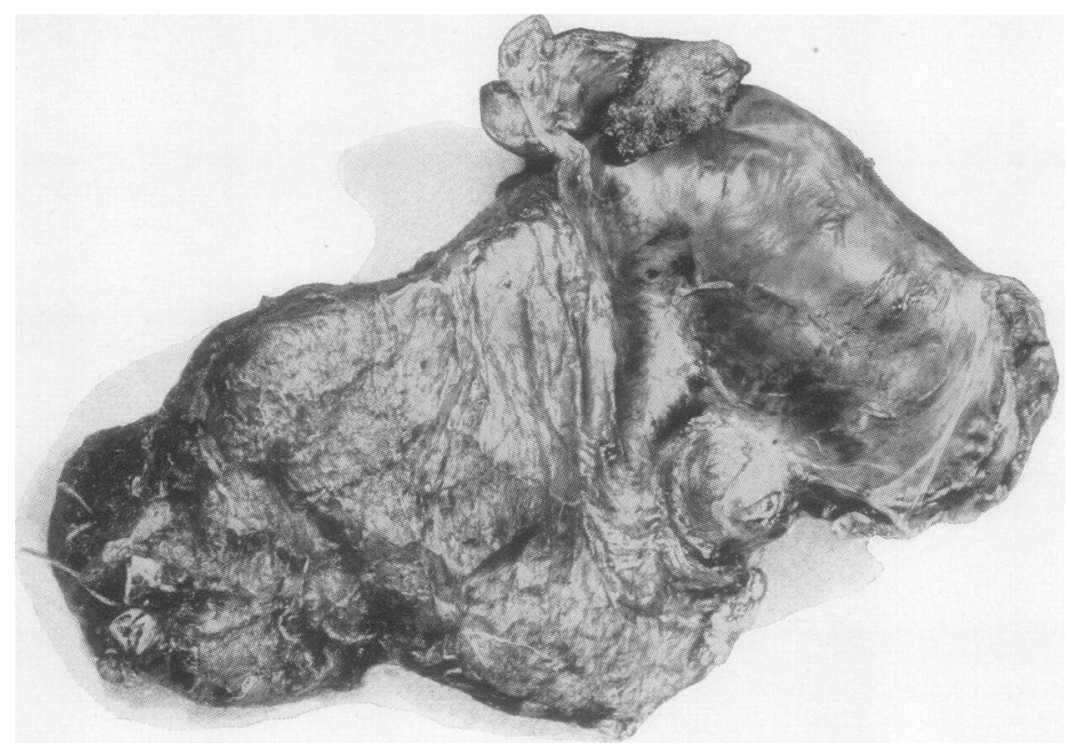

Fig. 5.-Left lateral view of the undissected specimen orientated as it lay in the body. The large peripheral sac is clearly seen.

Discussion
Pathological Anatomy and Terminology.-The dissection of our specimen showed that the major iesion consisted of a thinwalled, dilated, elongated, and tortuous pulmonary arterial branch opening widely into a single large peripheral cavity which in turn drained by wide openings into two pulmonary veins. In spite of its huge size, the communicating cavity between artery and veins occupied the same position in relation to these as do the capillaries which are present in a normal lung. The arterial branch concerned in the lesion was abnormally wide, long, and thin-

It was found that the bronchus divided normally into branches to the usual upper, lateral, and lower segments of the lingula and that the normal of the two pulmonary arterial branches divided to supply the upper and lateral segments. The thin-walled, hugely dilated branch of the pulmonary artery was evidently the artery to the lower segment of the lingula, and small branches were discovered leaving it to supply this segment of the lung. It pursued a tortuous course to the periphery and the bulges of its curves had given the lobulated appearance to the lesion before the lobe was dissected. It terminated by entering the large peripheral sac. This sac was drained by both the lingular veins which ran in an almost straight course back to the hilum, receiving small tributaries from all three segments of the lingula. The vein walls were of approximately normal thickness, unlike the dilated artery whose walls were even thinner than those of normal pulmonary veins.

About halfway towards the periphery there was a small anastomotic branch about 1/16 in. in diameter between the dilated pulmonary artery and the lower of the two veins. No other direct arteriovenous anastomoses were found.

When the dilated vessels and the anastomotic sac were opened their inside walls were found to be smooth like the intima of normal blood vessels.

Sections of the wall of the dilated artery, one of the dilated veins, and the sac all appeared surprisingly similar, and consisted mostly of collagen fibres. Special stains showed very little muscle, and no elastic tissue could be identified in the sections examined. walled, from its origin to its termination in the peripheral cavity ; but the veins, apart from their unusual origin in the sac and their increased diameter to accommodate the greater volume of blood flowing through them, showed less variation from normal. Their walls were of normal thickness, and their length only slightly increased.

No other specimen of this condition seems to have been dissected in this way, and it is doubtful whether this arrangement of the vessels has always been appreciated. Usually the portion of the lung containing the lesion has been cut across, and the section has shown a series of vascular spaces of various sizes which have been mistaken for the vascular spaces in a haemangiomatous tumour. It is easy to see that a section of our specimen cutting through the peripheral cavity and the irregular loops of the tortuous pulmonary artery would have produced the appearance of multiple vascular spaces exactly as pictured in the reports of other writers, and we submit that careful dissection of their specimens would have shown an arrangement similar to that found by us.

It is well known that the large lesions are often multiple, but the fact that many small dilated vessels are scattered through the adjacent lung tissue has not been generally recognized; only Whitaker (1947) seems to have noticed them. He described a cluster of large vessels up to one centimetre in diameter in a lobe he removed (probably the tortuous pulmonary artery cut several times 
in the section), and also many others about two millimetres in diameter scattered through the rest of the lobe. We found similar small dilated vessels in a!l the broncho-pulmonary segments of the lingula of our patient, but they were more numerous in the lower segment whose artery was concerned in the formation of the main lesion (Figs. 8 and 9). It is therefore clear that the large arteriovenous communication is not a circumscribed condition as at first sight it appears to be, but a localized exaggeration of a more diffuse process.

As far as can be judged without serial section, the small dilated vessels scattered in the lung tissue are dilated pulmonary arterioles and their communications with corresponding venules. They appear to be comparable to

FIG. 7.-Diagram to scale (in inches) of lateral view of the vessels and bronchi shown in Fig. 6. A, Normal pul. monary artery and bronchus to upper segment. B, Normal pulmonary artery and bronchus to lateral segment. $C$, The upper of the pul monary veins. $\mathrm{D}$, The large saccular arteriovenous com. munication. E, The lower of the pulmonary veins. $F$, The huge tortuous pul monary artery to the lower segment. G, The bronchus to the lower segment.

The diagram shows how the artery enters the sac which is drained by the two veins. The other small communi cation between the artery and the lower vein about twothirds of the way towards the periphery was not visible in this view. A diverticulum halfway down the artery is seen. A small branch ran from this into the lung substance. This and all the other pulmonary branches of the artery and tributaries of the veins which were all small were divided in order to dissect the main vessels free.

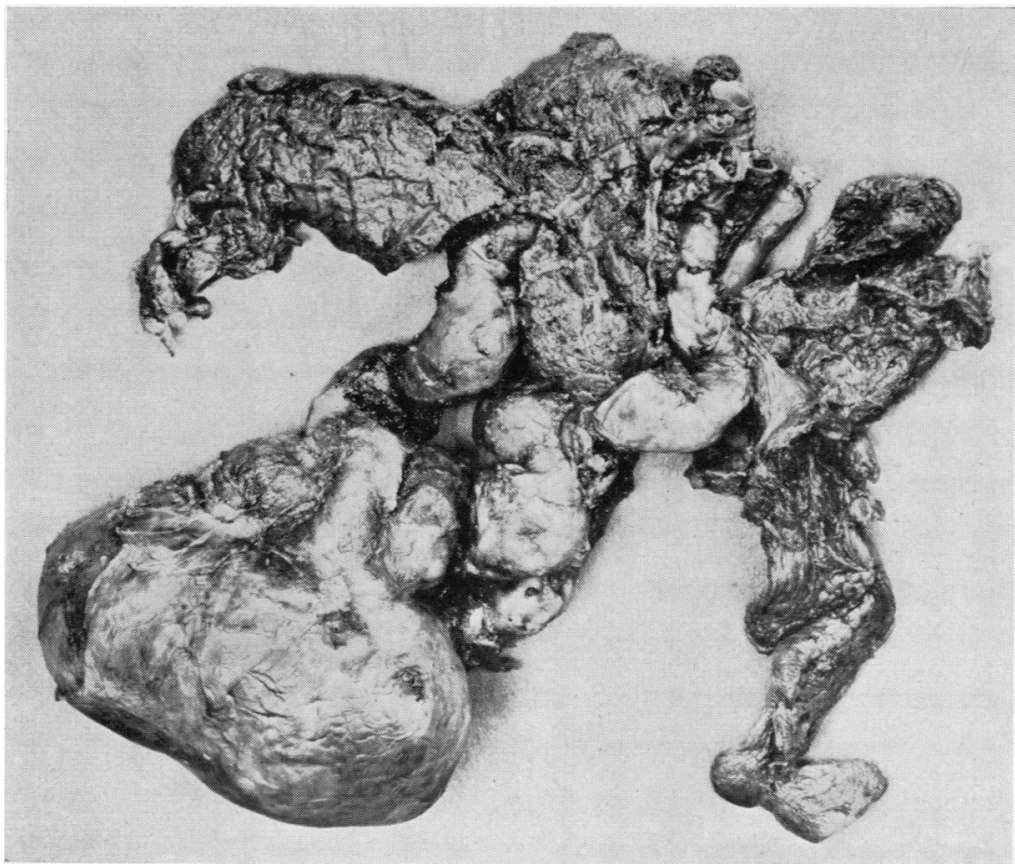

FIG. 6.-Same view of specimen (Fig. 5) after dissection. The upper segment of the lingula is reflected upwards, and the lower segment downwards. The lateral segment has been partly pushed between the artery and the upper vein so as not to overlie and obscure these vessels, which are therefore pushed a little further apart than they actually were.

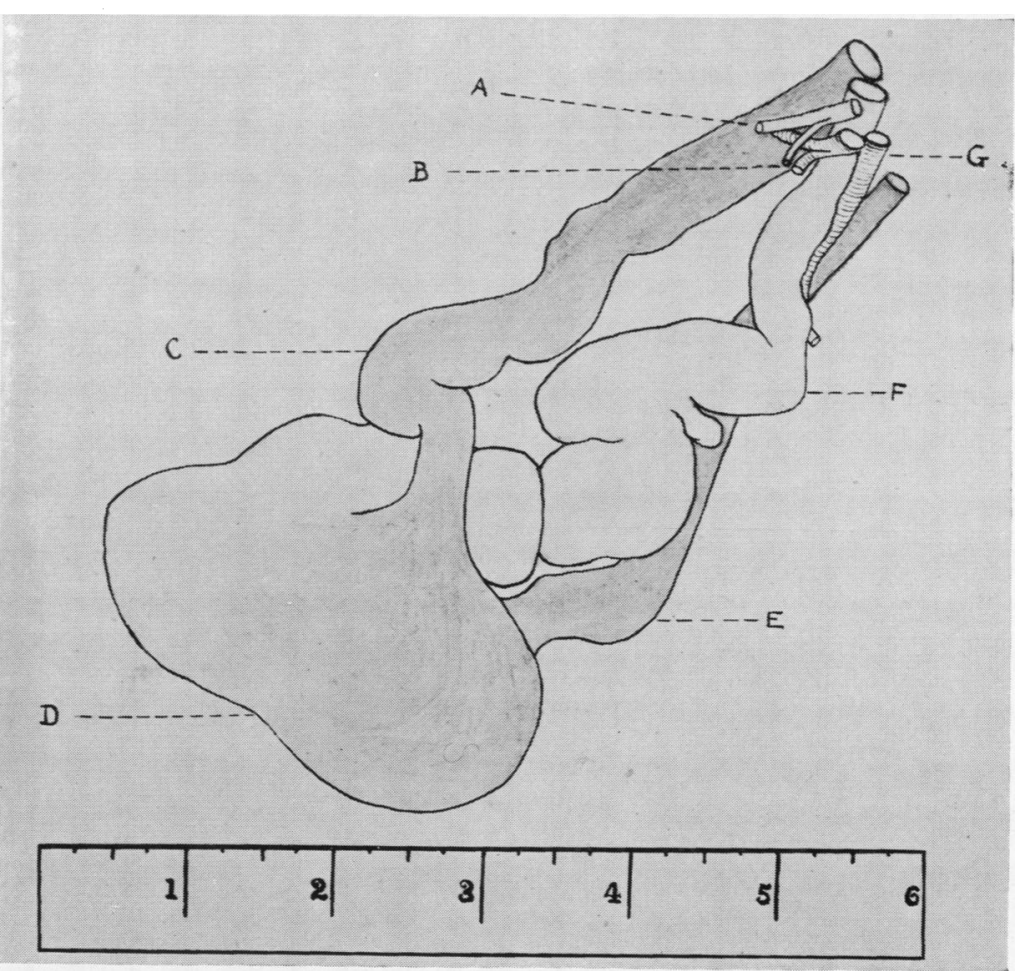


telangiectases in the skin and mucous membranes in which the red spot is produced by the blood in a dilated arteriole and its communications with venules. Moreover, the word telangiectasis means, by derivation, dilatation of a peripheral vessel. It is therefore reasonable to call the small dilated vessels scattered in the lung tissue in these cases pulmonary telangiectases.

It has already been pointed out that the large cavity in a pulmonary arteriovenous communication is, despite its size, merely a dilated peripheral vessel analogous in position to a capillary. Except for its size it is similar to small pulmonary telangiectases and to telangiectases in general. We have therefore decided to call these large cavernous arteriovenous communications in lungs cavernous pulmonary telangiectases.

We object to the term pulmonary arteriovenous fistula. The word fistula suggests to us an abnormal passage created by trauma or necrosis rather than a communication resulting from a developmental anomaly ; and the name pulmonary arteriovenous fistula implies a close analogy with the systemic arteriovenous fistula between large vessels usually caused by trauma, such as a gunshot wound. Both are large arteriovenous communications, but here the similarity ends. Their other features are in vivid contrast, and are conveniently stressed by being set out together.

A cavernous pulmonary telangiectasis is a huge peripheral vessel in the distal capillary bed. A systemic arteriovenous fistula is a true fistula which occurs proximal to the capillary bed supplied by the artery and drained by the vein. The circulation in the part distal to the fistula is impaired, and this may lead to oedema, varicosities, and eczema. There is nothing comparable to this in the lung.

The blood which flows through the arteriovenous communication in the lung by-passes the normal capillaries and is not oxygenated. Maier and his colleagues (1948) found that $58 \%$ of the total cardiac output at rest passed through the communication in their case. The stimulus of the resulting anoxaemia in the systemic circulation causes an increase in the circulating red cells. The blood plasma is not increased. Blood counts therefore show polycythaemia, and the increase in blood volume which is found is due to the increase in red cells. On the other hand, a patient with a systemic arteriovenous fistula has a raised blood volume due to an increase in the blood plasma. The stimulus which brings this about is the reduction in peripheral resistance which evokes the same response as a severe haemorrhage (Holman, 1937).
When a cavernous pulmonary telangiectasis is excised the normal red cell count is restored by increased blood destruction. In our patient the serum bilirubin was still raised (2.5 units) three weeks after operation. When a systemic arteriovenous fistula is excised the effect is like an autotransfusion, and the overdistension and high blood pressure are promptly rectified by a reduction in total blood volume through loss of blood plasma, shown by an excessive urinary output and by a concentration of the blood in the first 24 hours after the operation (Holman, 1944).

The proximal spread of dilatation along the artery involved in a cavernous pulmonary telangiectasis is limited. It has never been observed to reach to the right heart which remains normal in size. In a patient with a systemic arteriovenous fistula there is dilatation of the whole fistulous circulation which is bounded by the heart, the artery between the heart and the fistula, the fistula and the vein between the fistula and the heart; this circuit Holman describes as being parasitic on the normal circuit. Also, if the fistula is greater in diameter than the artery from which it opens " arteries in the collateral bed surrounding and proximal to the fistula will open up to pour their quota into the parasitic circuit to satisfy, as it were, its thirst for blood" (Holman, 1944). A cavernous pulmonary telangiectasis may, as in our case, be far wider than the artery which supplies it, but no collateral vessels pour blood into it.

By cardiac catheterization in their case of cavernous pulmonary telangiectasis, Maier and his colleagues (1948) found that the output of the right heart was within normal limits, and that the pressures in the right auricle, right ventricle, and pulmonary artery were normal. It would seem that in the pulmonary circulation there can be such satisfactory compensation for a large pulmonary arteriovenous communication drawing to itself over half the pulmonary blood flow that there is no appreciable effect on the mechanics of the right heart and pulmonary circulation. Most of the secondary effects of a systemic arteriovenous fistula are due to a lowering of peripheral resistance which it produces.

Enough has been written to show that cavernous pulmonary telangiectasis and systemic arteriovenous fistula are two utterly different conditions. The name pulmonary arteriovenous fistula for the former is unsatisfactory because it hints at a similarity between them which has no basis on fact and because it uses the word fistula in a misleading sense.

In addition, however, to systemic arteriovenous fistulae of the type we have been discussing, there 
are peripheral lesions of systemic vessels which do resemble cavernous pulmonary telangiectasis in many respects. They also are lesions in which large vascular, as distinct from fistulous, communications develop between dilated peripheral systemic arteries and veins. It is difficult to refer to them because here, again, the terminology is confusing. They are variously called cirsoid aneurysms (especially when in the scalp), congenital arteriovenous fistulae or arteriovenous aneurysms (especially when in the extremities), and cavernous haemangiomata (especially when in solid organs such as the liver and brain). Exact descriptions of the vessels in these lesions are frequently omitted, but where any attempt has been made to describe them dilated, tortuous veins seem always to have been seen. The arteries are frequently not mentioned, but when noticed have always been dilated, tortuous, and thin-walled (Israel, 1877 ; Reid, 1925 ; Horton and Ghormley, 1934). Presumably in these lesions the change in the arteries is less obvious than in the veins, but it is clearly similar to the

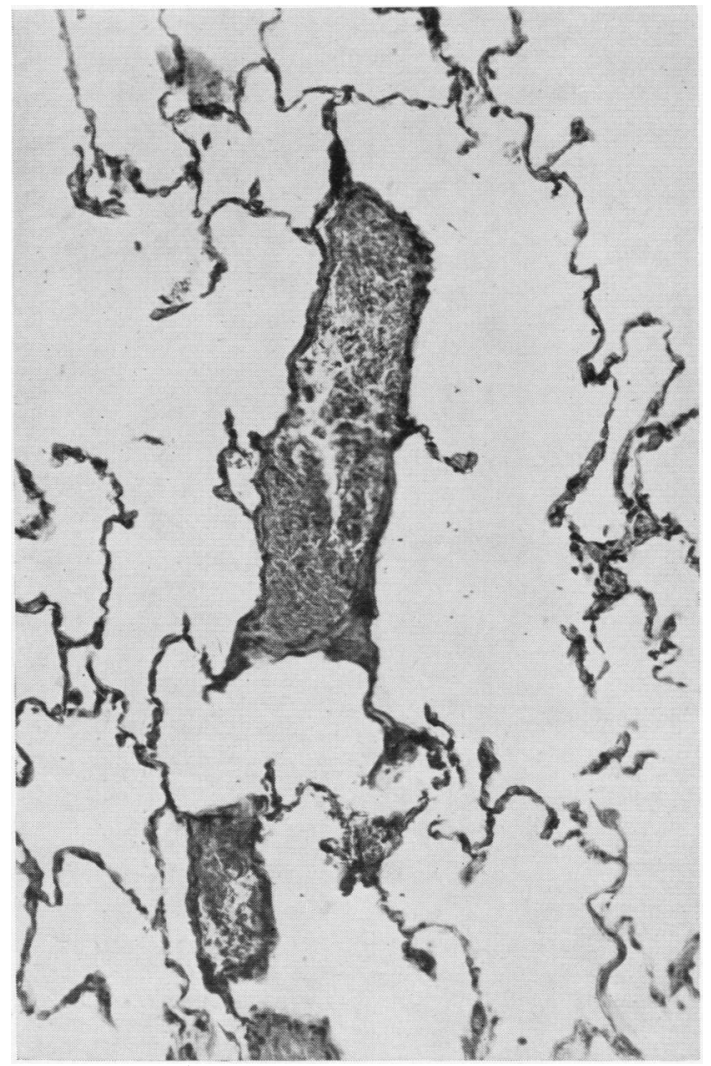

FIG. 8.-Photomicrograph of portion of lingula showing large thin-walled vessels in inter-alveolar walls. $\times 150$.

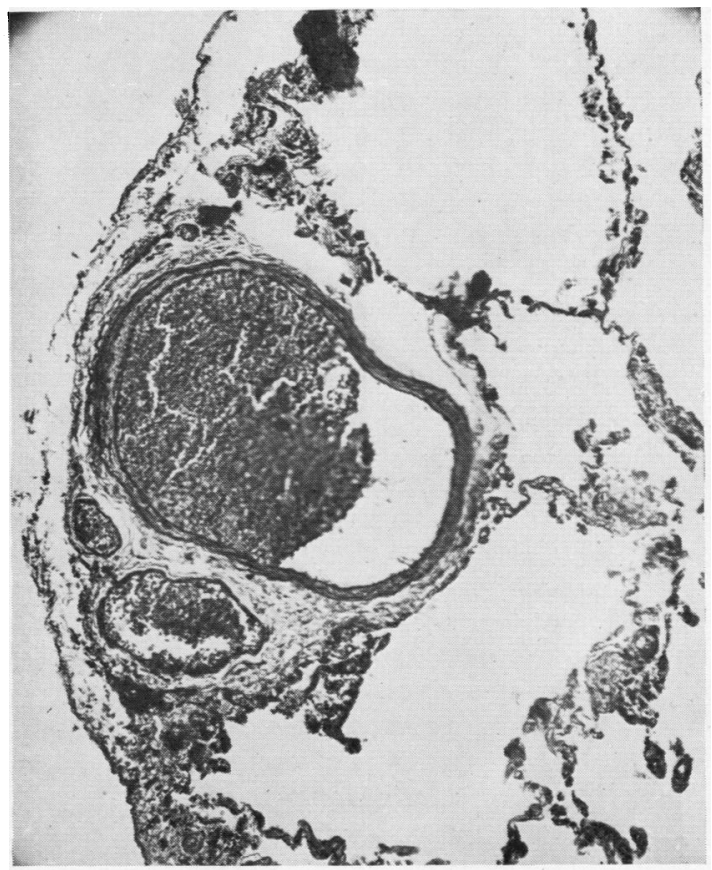

FIG. 9.-Photomicrograph of portion of lingula showing a large thin-walled vessel immediately beneath the visceral pleura. $\times 100$.

change in the pulmonary artery in the lesion in our patient. The arteriovenous communications in the systemic lesions are usually multiple, and small compared with our pulmonary one which was nearly two inches in diameter. Possibly the very large size in the lung may be due purely to physical factors such as the negative intrapleural pressure and the tendency of any thin-walled pulmonary cavity to enlarge due to the centrifugal pull of the elastic lung round it.

Unfortunately we have not studied any of these peripheral systemic lesions personally, and can give no first hand information about them, but from the evidence available it seems that their pathological anatomy is very similar to that of cavernous pulmonary telangiectases, and that they, too, might well be called cavernous telangiectases. For convenience they will be referred to as such in the remainder of this paper.

Reid (1925) suggests that all so-called cavernous haemangiomas, wherever situated, are really arteriovenous communications with dilated and tortuous afferent and efferent vessels, and that the appearance of multiple blood spaces in sections of them is an illusion produced by multiple cross section of continuous channels. 
Holman (1937) states that the physiological effects of systemic cavernous telangiectases (which he calls congenital arteriovenous communications) are, as would be expected, the same as in the acquired type (arteriovenous fistulae), but they need to be numerous and big for the effects to be evident.

Our conception of the pathological anatomy of this curious group of vascular anomalies may be summarized as follows.

When a vessel between an arteriole and its corresponding venule increases in diameter forming a little arteriovenous communication the result is a telangiectasis. The communicating vessel is in the same relative position as the normal capillaries, and may even be regarded as a modified capillary. When the increase in diameter continues, making the arteriovenous communication larger and larger, the afferent artery and efferent vein also dilate more and more and tend to become tortuous, and this process seems gradually to spread proximally along them. The artery also becomes thin-walled in its dilated part. The result is a cavernous telangiectasis. The lesions occur in both the systemic and pulmonary circulations. Cavernous telangiectases in the systemic circulation have previously been called congenital arteriovenous fistulae, cirsoid aneurysms, and cavernous haemangiomata. Cavernous telangiectases in the pulmonary circulation have previously been called pulmonary arteriovenous fistulae and cavernous haemangiomata.

Clinical Relationships of the Disease.-Having shown, from the standpoint of pathological anatomy, what we believe to be the relationship of cavernous pulmonary telangiectasis to telangiectases in general, it becomes of interest to consider its position in relation to certain other similar diseases from the clinical aspect.

In addition to the major pulmonary lesion our patient had scattered telangiectases elsewhere, and these are an almost constant feature in the case descriptions in the literature. They have been described in the skin, nail beds, buccal and nasopharyngeal mucosa, stomach, liver, and the remainder of the lungs, and are so constant that a patient with cavernous pulmonary telangiectases is better regarded as suffering from telangiectasia of which the large pulmonary lesions are merely the most conspicuous part.

Another interesting observation is that this pulmonary condition is not infrequently associated with a family history of telangiectasia (Goldman, 1947 ; Whitaker, 1947). All we could elicit from our patient was the vague story of a brother being subject to epistaxes.
These features suggest a fairly close relationship with hereditary familial telangiectasis first accurately described by Osler (1901), but they are not the only features suggesting a relationship. Recurrent epistaxes are very common in Osler's disease, and headaches and even migraine (Campbell, 1944) have been described. Our patient suffered from all three of these symptoms. In addition Cappon (1945) described a case of hereditary haemorrhagic telangiectasis in which polycythaemia developed between bleeds, and he postulated overaction of the bone marrow due to the stimulus of recurrent haemorrhage to account for this. He does not mention whether a radiograph of his patient's chest was taken, and one wonders if this was done. It will be remembered that our patient showed no polycythaemia after a severe melaena. Perhaps if all cases with hereditary telangiectasia had chest radiographs some would be found to have cavernous pulmonary telangiectases.

Though cavernous pulmonary telangiectases are usually associated with scattered telangiectases

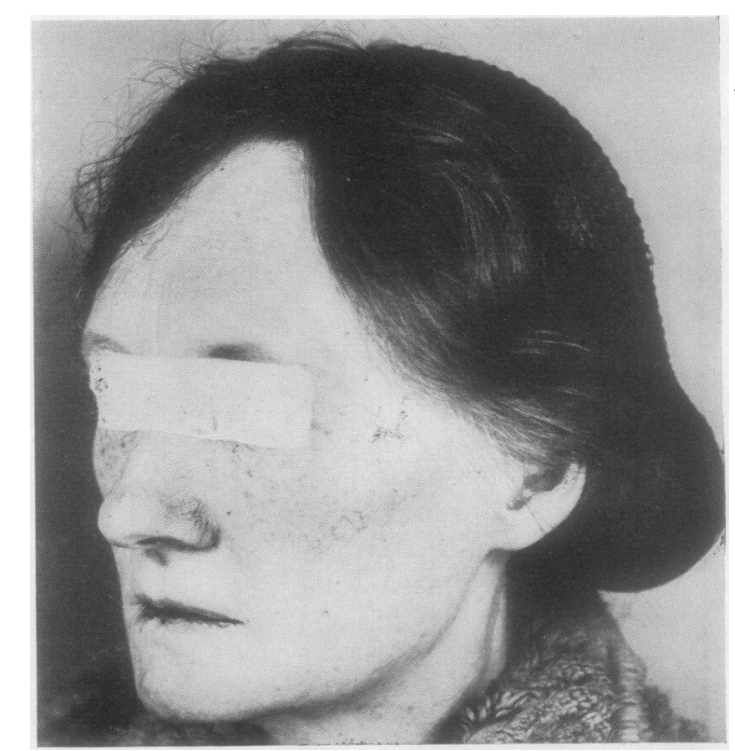

FIG. 10.-Photograph showing telangiectases which appeared on the patient's lower lip during preoperative period in hospital.

elsewhere, no author seems to have made a similar observation in cases with a systemic cavernous telangiectasis. The latter is therefore probably a much more localized maldevelopment.

Pathogenesis.-In none of these curious vascular anomalies is the aetiology understood, but it is worth while considering what little information is available to indicate possible causes. In the first 
place it is well known that telangiectases may be acquired. They may appear during pregnancy and in the course of certain diseases, and their development is characterized by fluctuation in size and number. They may even disappear completely. During our patient's stay in hospital two naevi were observed to appear on her lower lip (Fig. 10). They have the pulsatile centre and centrifugal blood flow described by Bean (1945) as characteristic of a cutaneous spider, so her case must be grouped with others in which these lesions may be acquired.

In such cases there is usually evidence of hormonal imbalance. Thus, in cirrhosis of the liver, there is known to be decreased destruction of oestrogens, and it is thought that the resulting increase may cause the telangiectases which are frequently seen in this condition. Bean (1945) showed by the injection of oestrogens in two cases of liver disease that the frequency and size of the telangiectases varied according to the dose administered. Naevi have also been described following the injection of oestrogens in the treatment of carcinoma of the prostate. The telangiectases which frequently appear during pregnancy recur in some individuals at the same stage of each gestation, and the occurrence of varicose veins and dilatation of the ureters in pregnancy is now thought to be due to the relaxing effect of increased oestrogens on plain muscle. The skin naevi may be a local manifestation of this effect on the musculature of the blood vessels.

The premenstrual epistaxis which constantly occurred in our patient points to some causative hormonal variation in her case.

We therefore believe that the evidence available justifies the suspicion that humoral factors play at least a part in the development of telangiectasia in general, including those cases with cavernous pulmonary telangiectases. We also believe that these factors may operate during life as well as before birth, and that there is little justification for regarding any of these diseases as certainly congenital. It is better to think of them as developmental anomalies which may take place in response to stimuli operating before or after birth, and even late in life. The clinical onset in most cases of telangiectasis, whatever the type, is long after birth.

In those showing hereditary and familial tendencies the inherited factor may be an increased tendency of the vessel walls to react in this way to certain stimuli, but here we enter the realms of speculation. Suffice it to say that we wish to stress the advantage of regarding these conditions as the local result of an abnormal humoral state. This conception is a stimulus to further investiga- tion, whereas classing them as tumours or congenital malformations tends, by creating the false impression that no more need be said or done about it, to close the mind to further inquiry. The urge to inquire is so easily inhibited that it is unwise to counter it with ill-chosen terminology.

It must be sufficiently apparent why we avoid the term haemangiomata for any of these conditions.

Management of Cavernous Pulmonary Telangiectasis.-Sufficient cases of cavernous pulmonary telangiectasis have been described for tentative suggestions about the management of this condition to be made. Excellent descriptions of the clinical syndrome associated with it have been given by Jones and Thompson (1944), Maier and his colleagues (1948), and others, and we have nothing to add to them. The syndrome is so characteristic that it has only to be known for the condition to be immediately suspected. Cyanosis, clubbing of the fingers, polycythaemia, and a general appearance suggestive of congenital cardiac disease in the presence of a normal heart should make one look for the typical shadow of a "tumour" in the lung radiograph If the shadow is present and its size increases during inspiration and decreases during expiration the diagnosis is virtually certain, though naturally all the other features of the syndrome will be looked for to complete the clinical picture. The point to be made here is that the correct diagnosis should usually be possible without exploratory thoracotomy.

Conservative treatment being of no value, the desision whether the lesion should be excised must then be made.

In the literature we have found records of 28 cases diagnosed during life, our case making the twenty-ninth. Of these 21 have been submitted to operation with two deaths (see Table).

Surgery is seen to have been frequently resorted to without undue risk, but the decision to operate should nevertheless be given careful thought. Facts

TABLE

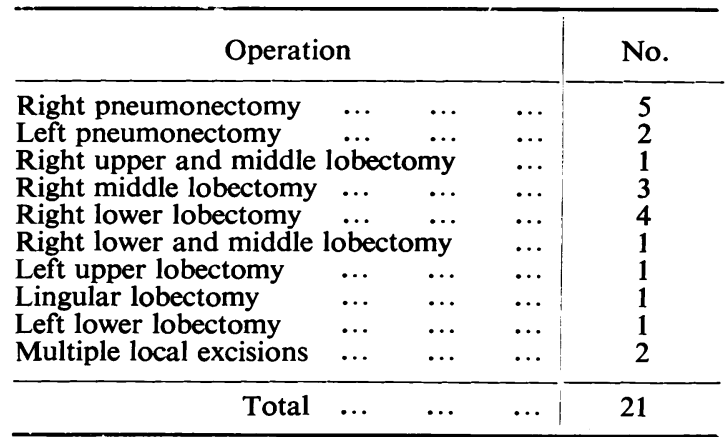


which should give pause are the knowledge that the cavernous lesion is merely the most conspicuous abnormality in a patient with telangiectasia, that the cavernous lesions may be multiple and bilateral, and that on more than one occasion the state of the blood vessels at the hilum discovered at operation has been such that pneumonectomy has been required for a lesion which preoperatively might have been expected to require a much less radical operation. Moreover we have as yet no certain evidence whether the arteriovenous communications in the cavernous lesions go on increasing in size (though presumably they do), and if so how fast, or whether fresh ones may appear after the successful removal of the first.

On the other hand we do know that the anoxaemia caused by a large arteriovenous communication in the lung is a serious handicap in life, and that the risk of fatal haemorrhage exists. Also Maier and his colleagues (1948) were of the opinion that subacute bacterial endarteritis had been present in the lesion in their case, so that this must be regarded as another possible complication.

Since insufficient cases have been described for the incidence of these complications to be known, we believe that too much importance should not be attached to them, and that the main indication for operation should be the degree of anoxaemia and its secondary effects such as polycythaemia. When the patient has obvious disabilities from this cause and it is judged that the disabilities will be largely relieved by operation, there is every justification for advising it. We did this with our patient even though it is probable that she has another small cavernous telangiectasis in her other lung. The clinical improvement has amply proved the wisdom of this course in her case.

\section{SUMMARY}

A case of cavernous pulmonary telangiectasis successfully treated by excision is described.

The pathological anatomy of the arteriovenous communication in this case has been shown by careful dissection in a way, as far as we know, not previously done.

The lesion has been compared and contrasted with arteriovenous communications of various types elsewhere in the body, and has been shown to differ from telangiectases only in size. It has therefore been called a cavernous telangiectasis, and our reasons for preferring this name are given.

It has been pointed out that it is merely the most conspicuous feature of a more widely distributed telangiectasia whose similarities to hereditary familial telangiectasis are indicated.
The probability that humoral factors play a part in its causation is suggested, and the fact that it is not necessarily congenital, and almost certainly not neoplastic, is stressed.

It is considered that the main indication for excision of the lesion should be the degree of disability resulting from the anoxaemia and its secondary effects.

This patient was referred to us by Dr. Margaret Henderson. We are indebted to the special departments of the Royal Melbourne Hospital for the various investigations. We wish particularly to thank the hospital pathologist, Dr. E. S. J. King, for his very great help and advice, and the hospital photographer, Mr. R. Inglis, for the illustrations.

\section{REFERENCES}

Adams, W. E. (1944). J. thorac. Surg., 13, 371.

Alexander, W. S. (1945). N. Zealand med. J., 44, 180.

Barnes, C. G., Fatti, L., and Pryce, D. M. (1948). Thorax, 3, 148.

Bean, W. B. (1945). Medicine, 24, 243.

Bisgard, D. (1948). J. thorac. Surg., 17, 25.

Bockus H. L. (1944). Gastroenterology. Philp Saunders. Boerema, I., and Brilman, R. P. (1948). J. thorac. Surg., $17,705$.

Bowers, W. F. (1936). Nebraska med. J., 21, 55.

Byron, F. R. (1948). J. thorac. Surg., 17, 25.

Campbell, A. M. G. (1944). Lancet, 2, 502.

Cappon, D. (1945). Brit. med. J., 1, 440.

Cleland, W. P. (1948). Thorax, 3, 48.

Duvoir, M., Picot, G., Pollet, L., and Gaultier, M. (1939). Bull. Mém. Soc. mét. Hôp., Paris, 55, 596. Quoted by Whitaker (1947).

Goldman A. (1943). Dis. Chest., 9, 479.

(1947). J. Lab. clin. Med., 32, 330.

Hepburn, J., and Dauphinee, J. A. (1942). Amer. J. med. Sci., 204, 681.

Holman, E. (1937). Arveriovenous Aneurysm, Macmillan Co., New York.

- (1944). Austral. New Zeal. J. Surg., 14, 82.

Horton, B. T., and Ghormley, R. K. (1934). Collected Papers of the Mayo Clinic, 26, 629.

Israel, J. (1877). Arch. f. klin. Chir., 21, 109. Quoted by Holman (1937).

Janes, R. M. (1944). Brit. J. Surg., 31, 270.

- (1944). J. thorac. Surg., 13, 372.

Jones, J. C., and Thompson, W.P. (1944). Ibid., 13, 357. Lindgren, E. (1946). Acta radiol. Stockh., 27, 585.

Maier, H. C., Himmelstein, A., Riley, R. L., and Bunin, J. J. (1948). J. thorac. Surg., 17, 13.

Makler, P. T., and Zion, D. (1946). Amer. J. med. Sci., 211, 261 .

Osler, W. (1901). Johns Hopk. Hosp. Bull., 12, 333.

Reid, M. R. (1925). Arch. Surg., 10, 601, 996. (1925). Ibid., 11, 25, 237.

Rodes, C. B. (1938). J. Amer. med. Ass., 110, 1914.

Rundles, R. W. (1945). Amer. J. med. Sci., 210, 76.

Samson, P. C. (1948). J. thorac. Surg., 17, 23.

Shefts, L. M. (1948). Ibid., 17, 23.

Shenstone, N. S. (1942). Ibid., 11, 405.

Smith, H. L., and Horton, B. T. (1939). Amer. Heart J., $18,589$.

Sweet, R. H. (1948). J. thorac. Surg., 17, 23.

Watson, W. L. (1947). Surgery, 22, 919.

Whitaker, W. (1947). Thorax, $2,58$. 